\title{
Considerations of Transient Electromagnetic Forces in Structural Behaviors for ITER Shield Blanket Components*
}

\author{
Alice Ying ${ }^{1}$, Joseph Kotulski ${ }^{2}$, Hongjie Zhang ${ }^{1}$, Mike Ulrickson ${ }^{2}$ \\ ${ }^{1}$ Mechanical and Aerospace Engineering Dept., UCLA, Los Angeles, CA 90095, USA \\ ${ }^{2}$ Sandia National Laboratories, Albuquerque, NM, 87185-1129, USA \\ ying@fusion.ucla.edu
}

\begin{abstract}
The structural designs of the ITER FW/shield blanket components take into account proper dissipation of the transient electromagnetic (EM) forces, and thus reduce the impact of those forces. In this paper we analyze how the blanket/electrical strap components react to $\mathrm{EM}$ forces and evaluate whether the design complies with the SDC-IC criteria under the envisioned fault conditions. Specifically, analyses were performed to evaluate the fatigue lifetime of the US proposed solid electric strap designs under a combined mechanical displacement and EM transient load. The stress analysis was performed using dynamic and time-independent static analysis. These results show that the EM forces tend to bend the strap away from its attachment points and lead to a large plastic strain, which results in a relatively short lifetime, and fails to meet ITER requirements. The study also shows that it is necessary for the analysis to take into account the time evolution of the EM force to correctly estimate the anticipated strain range. *The views and opinions expressed herein do not necessarily reflect those of the ITER Organization.
\end{abstract}

Keywords-electromagnetic forces; blanket/electrical strap; strain range; transient structural analysis

\section{INTRODUCTION}

The structural designs of the ITER FW/shield blanket components take into account proper dissipation of the transient electromagnetic (EM) forces and thus reduce the impact of those forces. For example, electrical straps are being used to electrically join the FW and the SB, and the SB and the vacuum vessel to dissipate the current. In an ITER IO design [1], the straps are formed and louvered from two sheets of $\mathrm{CuCrZr}$ alloy to achieve flexibility in all three directions. However, this electrical strap design has severe stress problems and resulted in a predicted fatigue lifetime of 3 to 300 cycles with one half the expected load applied (3000 cycles are needed at full load) [1]. To help identify proper electrical strap design, the US ITER FW/shield design team has proposed several variances [2] to the existing ITER IO electric strap design for exploratory, scoping analysis. In the proposed design, the team uses a solid copper sheet with a larger corner radius to replace the existing louvers and eliminates key slots as seen in the IO design (Figure 1). The copper sheet was bent to form a U-shaped configuration and was bolted into place through access holes. The proposed designs preserved the access holes for the leg clamp blocks. In this paper, analysis has been performed to evaluate the fatigue lifetime of the proposed electrical strap designs taking into account both mechanical faults and EM loads. A particular emphasis is on using dynamic analysis, as opposed to time-independent static analysis, in order to reveal EM transient time effect on the fatigue lifetime. The EM load is also applied to SB module 15 to study whether a wing-like construction can be impacted by the torque created by EM load.

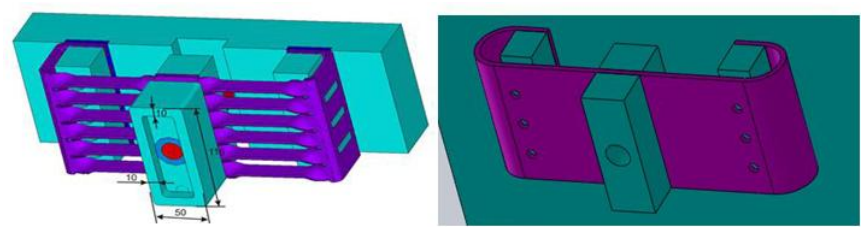

Fig. 1. Electric strap designs using louvers as in IO and solid sheets as proposed by the US team.

The proposed US solid strap is formed from a $5 \mathrm{~mm}$ thick sheet of CuCrZr-IG treatment A alloy. During the preliminary study, mechanical analysis was performed for several variances for scoping purposes. They vary among the size of the corner radius, the size of the bolt access hole, the length and the thickness of the strap. With each option, elastic analysis involved an imposed displacement of $+/-1$ $\mathrm{mm}$ in the toroidal plane to the center attachment and a fixed boundary to the leg attachment. The results from the elastic and elasto-plastic analysis showed that the most stress/strain was concentrated along the bend, while increasing the corner radius helped reduce its maximum magnitude. However, the thicker the strap the higher maximum strain is when the strap is imposed by the same displacement load. Furthermore, reducing the size of the bolt access hole removed the stress concentration found in the circumference of the bolt access hole. The preliminary analysis has identified a strap with a corner radius of $27 \mathrm{~mm}$, a bolt access hole of $9 \mathrm{~mm}$, and a strap length of $23 \mathrm{~mm}$ for further mechanical study. In addition, it was found that the aforementioned boundary condition led to a too rigid a boundary condition which prevented the strap from bending when the EM forces were applied. In the later analysis, a revised boundary condition, that incorporated a rigid body behind the strap allowing the strap to bend according to the applied EM forces, was incorporated, which is illustrated in Figure 2. The model used frictional contacts for all contacting surfaces between 
materials except where the two flat surfaces touch the rigid body. At that location, they were modeled as surface contact.

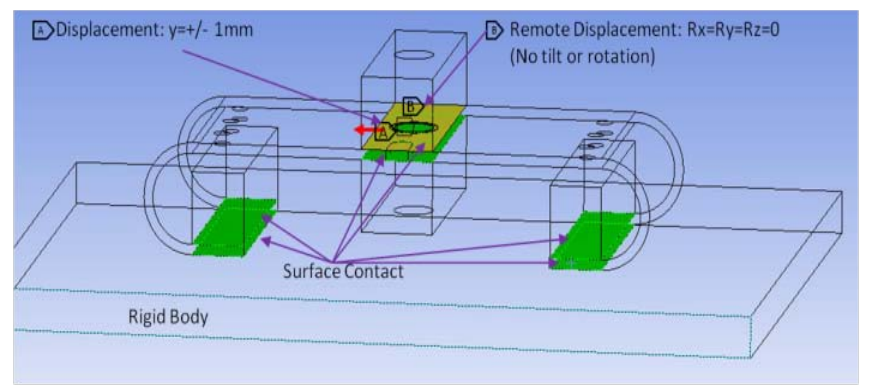

Fig. 2. Boundary conditions shows relative displacement of $+/-1 \mathrm{~mm}$ and no tilt or rotation in the toroidal plane to the center attachment and surface contacts to the leg attachment.

\section{IMPOSING EM FORCES FROM DISRUPTION CURRENT ANALYSIS FOR MECHANICAL ANALYSIS}

The volumetric EM forces were derived in a two-step process. First the halo-current distribution was calculated using the Opera-3d software, and then this current was coupled with the magnetic fields in the device to calculate the elemental volumetric force. Then the volumetric forces were transferred to nodal forces of the structural elements for stress analysis. The overall process consisted of: 1) Generating a mesh for the stress analysis using the ANSYS modeling software. 2) Creating a table file with the locations of the element centroids for this mesh. 3) Importing this file to the OPERA post-processor and extracting the EM forces at these locations (element centroids) from a disruption analysis. The element force data is then distributed among the nodes associated with the element to give the loads for the stress analysis. 4) Importing the nodal force data for the stress analysis. As noted, the loads are extracted at appropriate time points that yield the maximum stress (or the highest force).

Figure 3 illustrates the applied EM forces in the $\mathrm{CuCrZr}$ strap body. The EM force appears concentrated on the bent area of the $\mathrm{CuCrZr}$ sheet. The magnitudes of the force components are listed in Table I, of which the forces are between -2.18 to $1.89 \mathrm{~N}$ in the $\mathrm{x}$-direction, 0.00 to $0.83 \mathrm{~N}$ in the $\mathrm{y}$-direction, and -4.87 to $0.00 \mathrm{~N}$ in the $\mathrm{z}$-direction. The applied EM total force in the $\mathrm{CuCrZr}$ strap body included $\mathrm{FX}=-240.14 \mathrm{~N}, \mathrm{FY}=6790.13 \mathrm{~N}, \mathrm{FZ}=-39770.76 \mathrm{~N}$.

The EM forces induced by the disruption event MD_UP_LIN in shield block are illustrated in Figure 4. As shown, the EM forces exerted to the wings (material cutout to accommodated manifold pipes) apper in two opposite directions. The applied EM total force in SB15 body included $\mathrm{FX}=-8860.75 \mathrm{~N}, \mathrm{FY}=5080.39 \mathrm{~N}, \mathrm{FZ}=2498.29$ $\mathrm{N}$. We performed the stress analysis to examine whether the torque would be excessive. The radial torque calculated from this disruption event revealed two different peaks, the largest at $8.2 \mathrm{~ms}$ (near thermal quench) and another at $30 \mathrm{~ms}$ (during current quench). The stress range is between 10 and $25 \mathrm{MPa}$ in the regions of interest. These regions are away from the flexible mount locations where there are deep channels needed for other machine functions. Sm for this material is $127 \mathrm{MPa}$ so that the stress results are not as significant as we initially thought and are well below the failure criterion.

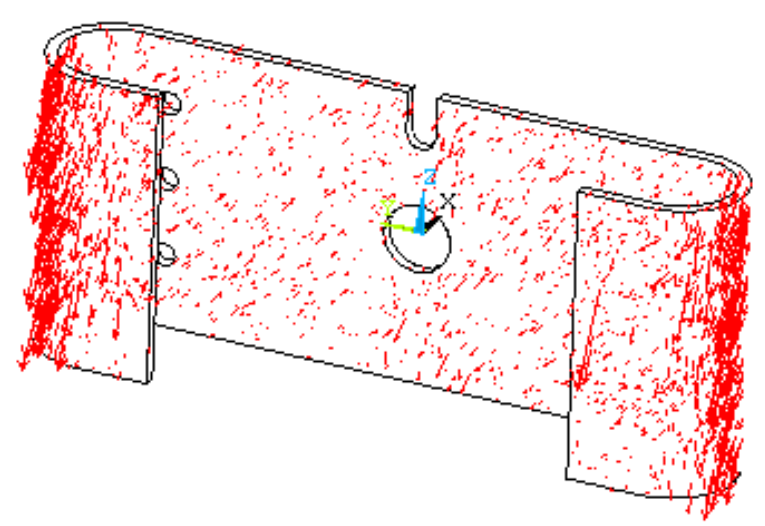

Fig. 3. The applied EM forces in the CuCrZr strap body.

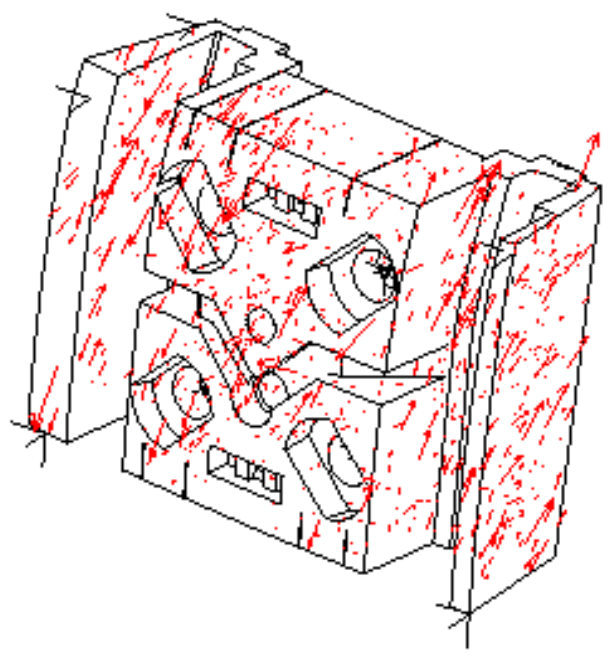

Fig. 4. The applied EM forces in the SB15

TABLE I. The MAGNITUdES OF THE EM ForCE COMPONENTS

\begin{tabular}{|c|c|c|c|c|}
\hline & & $\begin{array}{c}\text { The Whole E- } \\
\text { Strap }\end{array}$ & $\begin{array}{c}\text { Cu layer of E- } \\
\text { Strap }\end{array}$ & SB15 \\
\hline \multirow{4}{*}{$\begin{array}{c}\text { FX } \\
\text { (N) }\end{array}$} & Min & -2.18 & -2.18 & -63.45 \\
\cline { 2 - 5 } & Max & 1.89 & 1.89 & 127.39 \\
\cline { 2 - 5 } & Total & -154.77 & -240.14 & -8860.75 \\
\hline \multirow{3}{*}{$\begin{array}{c}\text { FY } \\
(N)\end{array}$} & Max & -0.026 & 0.00 & -63.48 \\
\cline { 2 - 5 } & Total & 11193.56 & 6790.13 & 86.06 \\
\hline \multirow{2}{*}{ FZ } & Min & -11.17 & -4.87 & -199.10 \\
\cline { 2 - 5 }$(\mathbf{N})$ & Max & 0.15 & 0.00 & 170.98 \\
\cline { 2 - 5 } & Total & -65562.25 & -39770.76 & 2498.29 \\
\hline
\end{tabular}




\section{TIME-INDEPENDENT AND TRANSIENT DYNAMIC MECHANICAL ANALYSIS FOR LIFETIME}

Mechanics analysis proceeded with a combined load of a mechanical fault of a $1 \mathrm{~mm}$ displacement in the toroidal plane and the EM induced forces for the optimized single layer $\mathrm{CuCrZr}$ electrical strap. A rigid boundary condition was used to simulate its bolted attachment (Figure 2). The analysis involved transient and time-independent static structural analysis to determine the dynamic response of the strap under the aforementioned loading conditions but oscillating between two opposite states. The idea was to evaluate how the response varies with time and how transient dynamics analysis differs from the time-independent static analysis. Four load steps were specified in the analysis as listed in Table II. In the transient analysis, an arbitrary time interval of $100 \mathrm{~s}$ was assigned to the time between the steps. (In next section, dynamic analysis is performed for a prototypical time interval of the applied EM forces.) For each step, the analysis was further divided into four substeps. ANSYS linearly interpolated the load between the steps for each sub-step.

TABLE II. CyClic LoAd VARIations In Four CyCles ANalysis

\begin{tabular}{|c|c|c|c|c|c|}
\hline Step & 0 & 1 & 2 & 3 & 4 \\
\hline Displacement (mm) & 0 & +1 & -1 & +1 & -1 \\
\hline EM Forces & 0 & $\mathrm{P}$ & $\mathrm{N}$ & $\mathrm{P}$ & $\mathrm{N}$ \\
\hline
\end{tabular}

The equation solved in the dynamic analysis is shown below [3]:

$$
[\mathbf{M}]\{\ddot{\mathbf{U}}\}+[\mathbf{C}]\{\dot{\mathbf{U}}\}+[\mathbf{K}]\{\mathbf{U}\}=\left\{\mathbf{F}^{a}\right\}
$$

Where $[\mathbf{M}]=$ structural mass matrix, $[\mathbf{C}]=$ structural damping matrix, $[\mathbf{K}]=$ structural stiffness matrix, $\{\ddot{\mathbf{U}}\}=$ nodal acceleration vector, $\{\dot{\mathbf{U}}\}=$ nodal velocity vector, $\{\mathbf{U}\}$ $=$ nodal displacement vector, and $\left\{\mathbf{F}^{\mathrm{a}}\right\}=$ applied load vector.

Since there is no obvious data on how much damping can be applied to the system, we ignored the damping effect during the transient dynamic analysis. For the end of each load step, the displacement plots are shown in Figure 5. The plastic strain plots at the end of the fourth step are shown in Figure 6. As shown, there is not much difference in the results between the transient and the static calculations. We learned that if the time-scale of the loading is such that the inertia and dumping effects are not important, a static analysis can be sufficient to determine the dynamic response. ANSYS obtains the nodal deformation at the end of each step using the current deformed information with reference to the initial nodal coordinate information. It is interesting to see that the maximum strain occurs at the location far from the maximum deformation location. Although it appears that a large deformation has occurred during loading, most locations with apparent deformation are in the elastic regime.

Under the applied cyclic loads, a significant plastic flow is induced near the maximum plastic strain location, close to the edge where the strap is attached to the rigid body (as shown in Figure 7). When the stress-strain tensor following the load steps is monitored at the location near the maximum

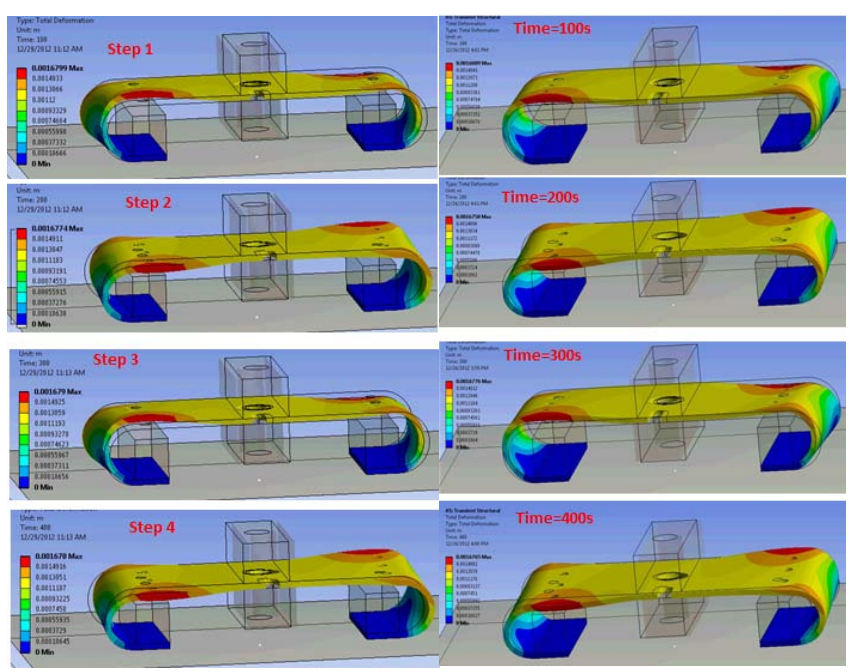

Fig. 5. Deformation characteristics of the electric strap subjected to a combined mechanical displacement of $1 \mathrm{~mm}$ and EM forces at the end of each load step (left): static elasto-plastic analysis; (right) transient elastoplastic analysis.

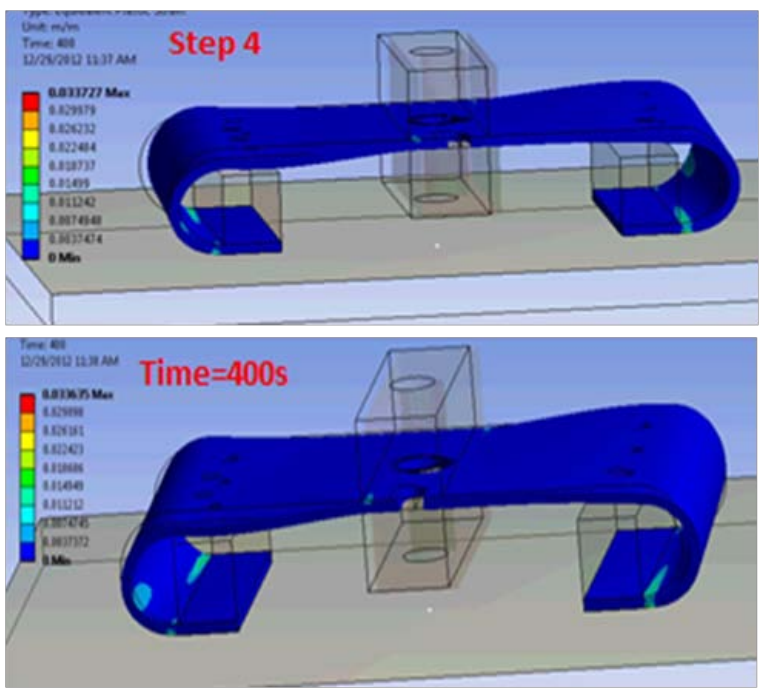

Fig. 6. Plastic strain characteristics of the electric strap subjected to a combined mechanical displacement of $1 \mathrm{~mm}$ and EM forces at the end of the $4^{\text {th }}$ cycle (top): static elasto-plastic analysis with a maximum of 0.033727 ; (bottom) transient elasto-plastic analysis with a maximum of 0.033635 .

plastic strain area, it reveals the hysteresis loop (Figure 8). Note that there is a linear portion of the stress-strain relationship which represents an elastic behavior. This is consistent with the true stress-strain data: as the strain approaches $0.144 \%$, it shows a plastic behavior. The hysteresis loop indicates that the strap is approaching hardening. As it approaches the next cycle, it requires higher stress for the same amount of strain, although the numbers of the cycles are not sufficient to offer a definitive answer. The hysteresis loop acts differently at different locations; when it is plotted for the point slightly away from the attachment edge, as shown in Figure 9, it shows a much larger displacement in the toroidal plane (ey). The hysteresis curve at the location where the deformation is the highest 
(see Figure 5) is shown in Figure 10. Even this location reveals the highest deformation; its behavior is more elastic and no component of the stress-strain tensor exhibits a hysteresis loop.

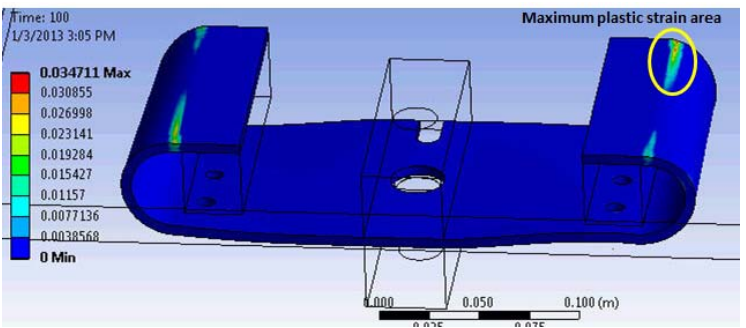

Fig. 7. Maximum plastic strain was found near the edge of the attachment using transient elasto-plastic analysis at the end of $1^{\text {st }}$ step.

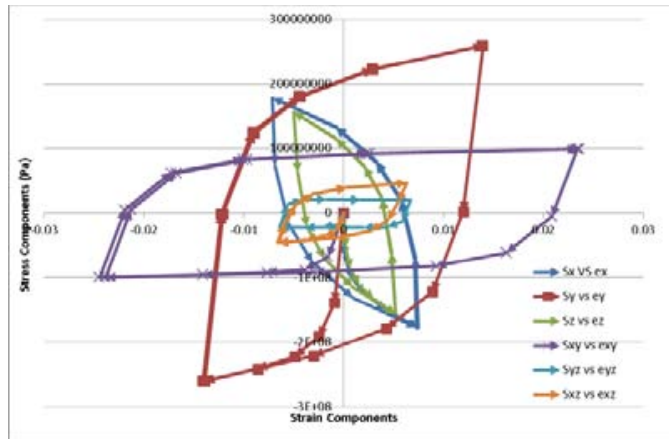

Fig. 8. Cyclic stress-strain curve at location near the edge of the attachment shows hysteresis effects ( $\mathrm{Sx}$ reads as stress $\sigma_{\mathrm{x}}$, ex reads as strain $\varepsilon_{\mathrm{x}}$ etc.)

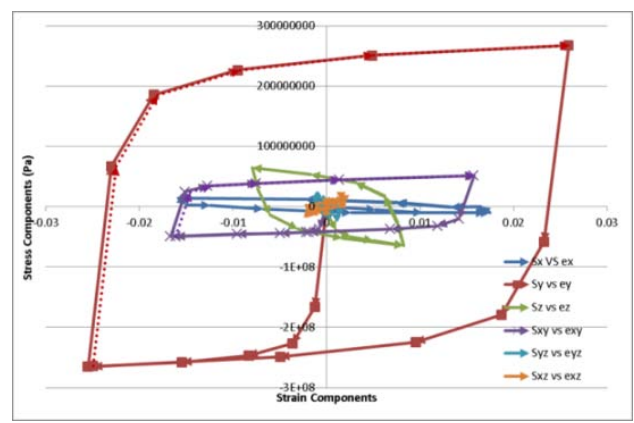

Fig. 9. Cyclic stress-strain curve at the location slightly away from the attachment line shows hysteresis effect (dotted lines are for the 4th cycle).

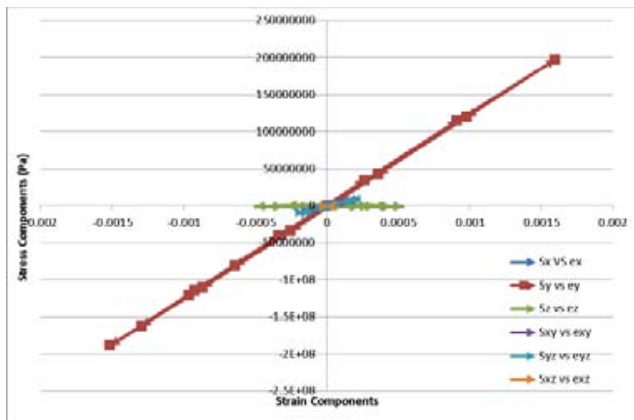

Fig. 10. Cyclic stress-strain curve at the location of maximum displacement shows no hysteresis effect.
The elastic and plastic strain tensors along the path near the maximum strain location for each step were extracted from the ANSYS calculations. These strain tensors were then used to calculate the equivalent strain range $\left(\overline{\varepsilon\left(t, t^{\prime}\right)}\right)$ between the steps (at $t$ and $t^{\prime}$ ) based on the difference between the strain range tensor components for each pair of instants $t$ and $\mathrm{t}^{\prime}$ of a cycle $\varepsilon_{x x}\left(t, t^{\prime}\right)$ as [4]:

$$
\begin{gathered}
\varepsilon_{x x}\left(t, t^{\prime}\right)=\varepsilon_{x x}(t)-\varepsilon_{x x}\left(t^{\prime}\right) \\
\overline{\varepsilon\left(t, t^{\prime}\right)}=\frac{\sqrt{2}}{3}\left\{\varepsilon_{x x}\left(t, t^{\prime}\right)-\varepsilon_{y y}\left(t, t^{\prime}\right)\right\}^{2}+\left[\varepsilon_{y y}\left(t, t^{\prime}\right)-\varepsilon_{z z}\left(t, t^{\prime}\right)\right]^{2} \\
\left.+\left[\varepsilon_{z z}\left(t, t^{\prime}\right)-\varepsilon_{x x}\left(t, t^{\prime}\right)\right]^{2}+6\left[\varepsilon_{x y}^{2}\left(t, t^{\prime}\right)+\varepsilon_{y z}^{2}\left(t, t^{\prime}\right)+\varepsilon_{z x}^{2}\left(t, t^{\prime}\right)\right]\right\}^{11 / 2}
\end{gathered}
$$

The equivalent strain is composed of elastic and plastic strain component for each cycle. The calculated total strain range between the cycles was about $5 \%$ for the assumed load variations (Table II). This implies that the fatigue lifetime of the strap is extremely short under this type of load cycling.

\section{EFFECT OF DAMPING}

The hysteresis curve at the location where the deformation is the highest (see Figure 4) is shown in Figure 9. Even this location reveals the highest deformation; its behavior is more elastic and no component of the stressstrain tensor exhibits a hysteresis loop.

The most common form of damping is the so-called Rayleigh type damping [5], where:

$$
[C]=\alpha[M]+\beta[K]
$$

and $\alpha$ is the viscous (or mass) damping component, and $\beta$ is the hysteresis or solid or stiffness damping component. In many practical structural problems, the $\alpha$ damping which represents friction damping may be ignored $(\alpha=0)$. In one parametric study, damping coefficients $\beta$ of $0,2 \%, 5 \%$, and $10 \%$ were considered to see how damping affected the structural behavior of the strap. In the first ten seconds, the load applied to the electrical strap includes only $1 \mathrm{~mm}$ displacement placed on the strap. The EM force kicks in at $10 \mathrm{~s}$ and reaches its full value at $10.01 \mathrm{~s}$. The EM force stays constant for $0.08 \mathrm{~s}$, and decreases to zero at $10.1 \mathrm{~s}$. After that, the strap is subjected to $1 \mathrm{~mm}$ displacement for another $10 \mathrm{~s}$.

The plastic strain increases after the EM forces are applied. The maximum plastic strain evolutions during the period for different $\beta$ values are displayed in Figure 11. As shown, the plastic strain approaches its asymptotic value before the EM force dissipates. Moreover, the evolution characteristics and the transient time to reach the saturated value depend on the value of $\beta$. Additionally, there is a larger decrease in the maximum plastic strain value at a higher $\beta$ value. The maximum plastic strain caused by the EM forces approaches the value calculated using zero damping if a $\beta$ value of $2 \%$ is used.

A similar behavior is observed for the case where the EM load only lasts for $0.04 \mathrm{~s}$ as seen in the VDE_DW_FAST event. We observe again a reduction in the plastic strain if the electric strap embodies some damping mechanism with a stiffness damping coefficient of more than $0.5 \%$ (Figure 12 ). 
In addition, there is a noticeable change in the strain evolution even with a $0.5 \%$ damping applied to the dynamic analysis. Since the damping coefficient depends on the frequency of the vibration mode of the body, we performed modal analysis to determine the vibration frequency of the electric strap under different modes of vibration. The lowest frequency indicates that the strap vibrates at $\sim 265$ cycles per second. However, it appeared difficult to estimate the damping coefficient without actual experimental test data from a vibration testing. As for a typical solid material, one expects a damping coefficient of $2 \%$ or less; therefore, we conclude that the effect of damping on the plastic strain reduction is not significant enough to increase the fatigue lifetime of the electric strap under the anticipated EM forces.

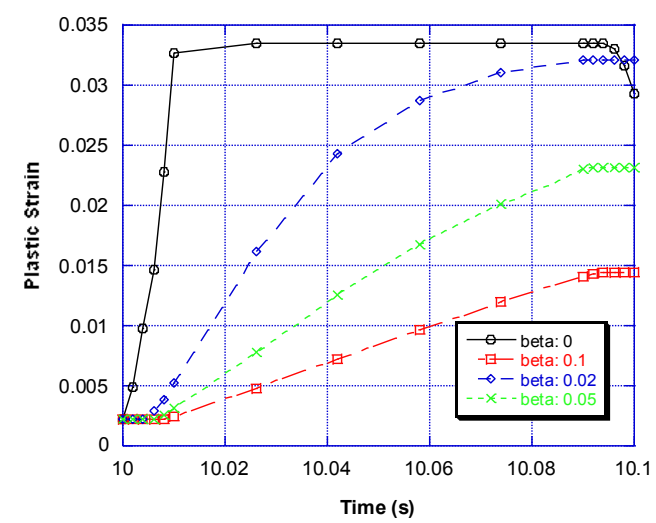

Fig. 11. Plastic strain evolution during the period when the E-strap encounters the electromagnetic forces for different damping coefficients $\beta$.

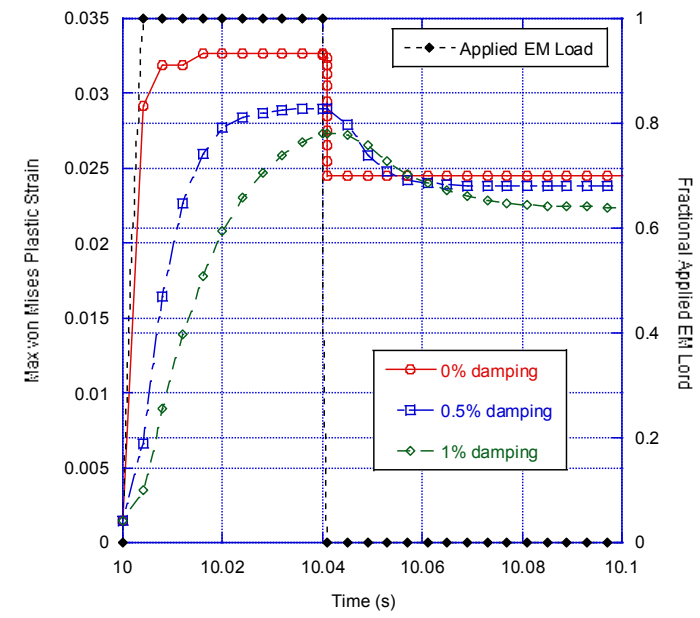

Fig. 12. Maximum von Mises plastic strain in the $\mathrm{CuCrZr}$ single layer electric strap as a function of time under different damping coefficients.

\section{EFFECT OF ForCE EVOLUTION CHARACTERISTICS}

Then we investigated the time effect of the applied EM forces on the mechanical behavior of the single layer $\mathrm{CuCrZr}$ electrical strap under a combined load of a mechanical fault of a $1 \mathrm{~mm}$ displacement in the toroidal plane and the EM induced forces. Specifically, while the mechanical displacement was applied at all times during the analysis, the EM force lasted only for the duration of time of the disruption event. Two disruption events were considered; one involving VDE DW FAST and the other VDE_DW_EXP. In each event, the EM force amplitude time evolution was scaled according to the EM current time evolution (The force is the largest at the highest magnitude of current). In the VDE_DW_FAST the EM force lasted for 0.04 seconds as shown in Figure 13, while in the VDE DW EXP the EM force lasted for 0.01 seconds as shown in Figure 14. Not only does the magnitude of the EM force vary with time, it also changes direction during the disruption event. At all times, a mechanical fault of $1 \mathrm{~mm}$ displacement in the toroidal plane was applied. The applied loads were repeated for three cycles (with a $10 \mathrm{~s}$ time interval between cycles), and for each cycle the EM forces were brought to their full values instantaneously (neither ramp-up nor ramp-down time). A damping coefficient of $0.5 \%$ was applied to each run to ensure dynamic analysis.

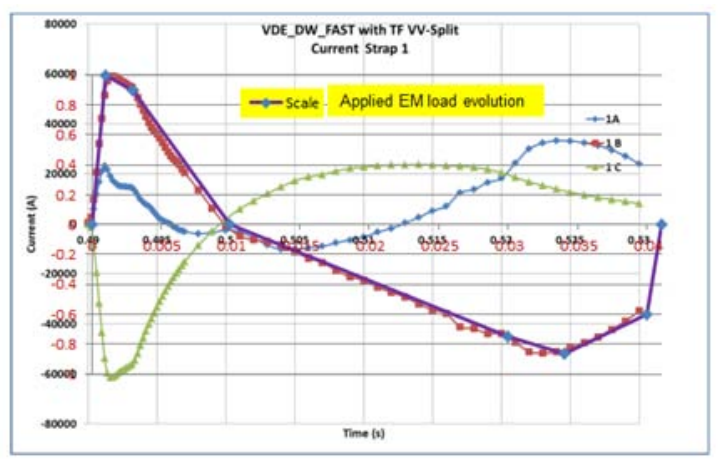

Fig. 13. Applied EM load time evolution scaled with the EM current time behavior under VDE_DW_FAST

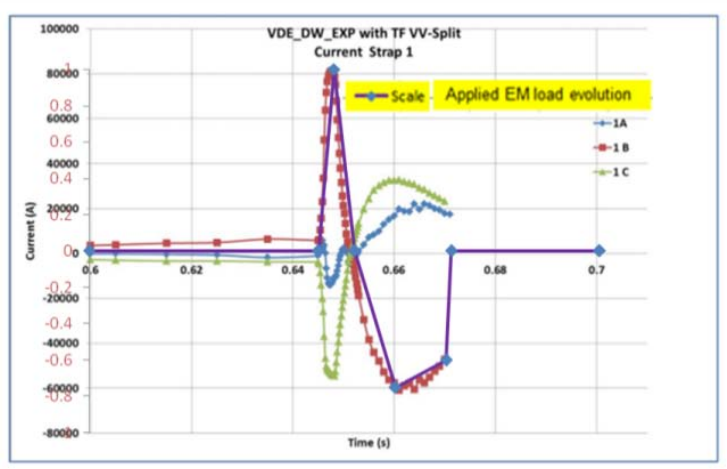

Fig. 14. Applied EM load time evolution scaled with the EM current time behavior under VDE_DW_EXP

The calculated maximum plastic strain evolution over the three cycles is shown in Figure 15 and compared with that of a constant EM force with the maximum amplitude applied over 0.04 seconds. As shown, the maximum strain was reduced from $2.38 \%$ to $1.878 \%$ for VDE_DW FAST and to $1.52 \%$ for the VDE_DW_EXP when the applied EM force was removed. However, the strain range caused by EM forces increased when the EM force evolution data, instead of constant force, was used as an input load. The change in the maximum strain was larger for the VDE_DW_FAST event than for the VDE_DW_EXP event. This might have 
been caused by the damping effect due to the amount of time the EM force oscillated is longer in the VDE_DW_FAST event.

The elastic and plastic strain tensors were extracted from the ANSYS calculations along the path near the maximum strain location for each case and at each cycle. These strain tensors were then used to calculate the equivalent strain range $\left(\overline{\varepsilon\left(t, t^{\prime}\right)}\right)$ between the cycles according to Eq. 2 and Eq. 3. Under the assumed fault loading conditions, the strain range varies between $0.92 \%$ for constant maximum EM force event, $1.34 \%$ for VDE_DW_EXP event, and 2.12\% for VDE_DW_FAST event. These strain ranges are lower compared to the values shown previously under the four cycles' fault loading conditions, in which not only did the EM forces oscillate between positive and negative directions with a maximum force magnitude, but the mechanical displacement also oscillated between $+/-1 \mathrm{~mm}$. These strain ranges resulted in fatigue lifetimes of 305,49 , and 13 cycles, respectively [6].

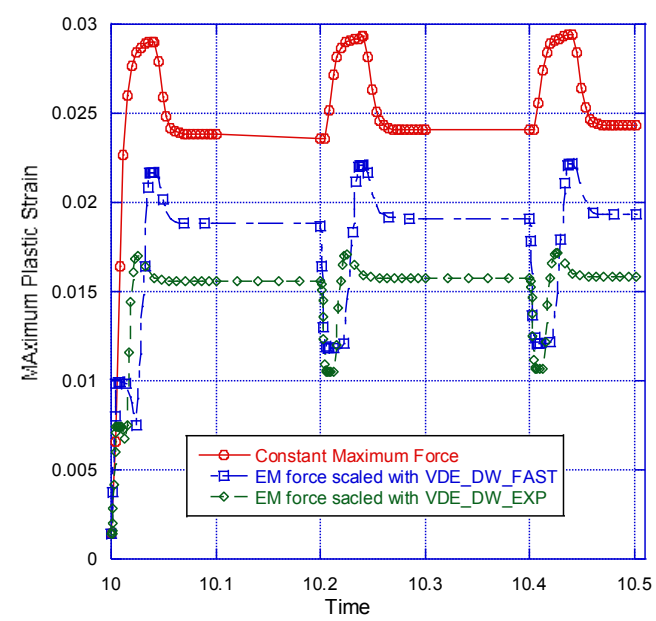

Fig. 15. Calculated maximum plastic strain evolution for three disruption events: constant maximum EM force, EM force scaled with VDE_DW_FAST and VDE_DW_EXP.

\section{SUMMARY}

The EM forces from a disruption event cause a significant increase in stress and plastic strain for the US proposed solid electric strap, which results in a short fatigue life under the assumed fault conditions. (A more complex shape of the electrical strap is being pursued in the ITER IO, which shows an acceptable fatigue lifetime.) This study shows that it is necessary to take into account the time evolution of the EM force into the structural analysis in order to correctly estimate the strain range. The cyclic strain range behavior can be analyzed using time-independent static analysis if the damping effect can be ignored. The estimated fatigue lifetime for the US ITER FW/Shield Design Team proposed electrical strap remains relatively short compared with the ITER required fatigue lifetime.

\section{ACKNOWLEDGMENT}

This work was jointly supported by the U. S. Department of Energy Contract DE-FG03-ER52123 and by grants from Sandia National Laboratories as part of the DOE's funded ITER project.

\section{REFERENCES}

[1] Ph. Chappuis, Electrical Straps Task Force: Shield Block to Vacuum Vessel, BIPT37, ITER IO, Sep. 2012

[2] M. Ulrickson, Electrical Strap Options, Aug. 6, 2012, US ITER Conference call

[3] ANSYS Manual, Ch. 5 Transient Dynamic Analysis, ANSYS 14.0

[4] ITER Structural Design Criteria for In-vessel Components, SDC-IC, Appendix B: Guidelines for Analysis. , IDM: ITER D222 HRC

[5] S. Hale, Damping in ANSYS/LS-Dyna, 2007 CAE Associates

[6] ITER Structural Design Criteria for In-vessel Components, SDC-IC, Appendix A: Materials Design Limit Data (2004, 2011), and Summary of material properties for structural analysis of the ITER internal components, ITER_D_23HL7T v.3.2, 2009. 